\title{
A QUANTITATIVE RIEMANN-LEBESGUE LEMMA WITH APPLICATION TO EQUATIONS WITH MEMORY
}

\author{
FILIPPO DELL'ORO, ENRICO LAENG AND VITTORINO PATA
}

\begin{abstract}
An elementary proof of a quantitative version of the Riemann-Lebesgue lemma for functions supported on the half line is given. Applications to differential models with memory are discussed.
\end{abstract}

\section{Statement of the Result}

Let $f \in L^{1}\left(\mathbb{R}^{+}\right)$be a real function, absolutely continuous on $\mathbb{R}^{+}=(0, \infty)$, and whose derivative $f^{\prime}$ is summable in a neighborhood of infinity (hence $f^{\prime} \in L^{1}(x, \infty)$ for every $x>0$ ). The aim of this note is to provide an elementary proof of the asymptotic expansion as $\lambda \rightarrow \infty$ of the half Fourier transform

$$
\hat{f}(\lambda)=\int_{0}^{\infty} \mathrm{e}^{-\mathrm{i} \lambda s} f(s) \mathrm{d} s
$$

depending only on the behavior of $f$ in a neighborhood of zero, establishing a quantitative version of the Riemann-Lebesgue lemma for functions supported on the half line. The analogue for the Laplace transform is the well-known initial value theorem (see e.g. [11]). For the Fourier counterpart, however, we could not locate a precise reference, although similar results are mentioned in [3]. Such an asymptotic expansion turns out to be extremely useful, for instance, when dealing with differential equations containing convolution terms, as shown in the last part of the work.

Notation. For every $p \in[0,1)$, we define the number

$$
\mathfrak{n}(p)=-\mathrm{i} \mathrm{e}^{\frac{\mathrm{i} p \pi}{2}} \Gamma(1-p),
$$

where $\Gamma(x)=\int_{0}^{\infty} s^{x-1} \mathrm{e}^{-s} \mathrm{~d} s$ is the Euler Gamma-function.

Theorem 1. Let $p \in[0,1)$. Assume that

$$
\lim _{s \rightarrow 0} s^{p} f(s)=\ell \in \mathbb{R}
$$

and

$$
\limsup _{x \rightarrow 0} x^{p} \int_{x}^{\infty}\left|f^{\prime}(s)\right| \mathrm{d} s<\infty
$$

Then

$$
\lim _{\lambda \rightarrow \infty} \lambda^{1-p} \hat{f}(\lambda)=\ell \mathfrak{n}(p) .
$$

2010 Mathematics Subject Classification. 42A38, 37L15, 45K05.

Key words and phrases. Half Fourier transform, Riemann-Lebesgue lemma, equations with memory, semiuniform stability, decay rates. 
$\diamond$ If $p=0$, assumption (2) merely means $f^{\prime} \in L^{1}\left(\mathbb{R}^{+}\right)$. Since $f$ is summable, this is always the case when $f$ is monotone and (1) holds.

$\diamond$ If $p>0$, it is easily seen that (2) is satisfied whenever the function $s \mapsto s^{1+p} f^{\prime}(s)$ is bounded in a neighborhood of zero, or whenever $f$ is monotone in a neighborhood of zero and (1) holds.

\section{Proof of Theorem 1}

Two general lemmas will be needed.

Lemma 2. Let $p \in(0,1)$. Then for any $\lambda, \beta>0$, we have the equality

$$
\lambda^{1-p} \int_{0}^{\frac{\beta}{\lambda}} \frac{\mathrm{e}^{-\mathrm{i} \lambda s}}{s^{p}} \mathrm{~d} s=\mathfrak{n}(p)+\frac{\mathrm{ie}^{-\mathrm{i} \beta}}{\beta^{p}}-\frac{\mathrm{i} p}{\beta^{p}} \int_{1}^{\infty} \frac{\mathrm{e}^{-\mathrm{i} \beta s}}{s^{1+p}} \mathrm{~d} s
$$

Proof. Via complex integration of the function $g(z)=z^{-p} \mathrm{e}^{-z}$ along the first quarter of the circumference of radius $R \rightarrow \infty$ (removing a small portion about zero), one draws the equality

$$
\int_{0}^{\infty} \frac{\mathrm{e}^{-\mathrm{i} s}}{s^{p}} \mathrm{~d} s=\mathfrak{n}(p)
$$

Hence, splitting the integral and performing a change of variables, we are led to

$$
\lambda^{1-p} \int_{0}^{\frac{\beta}{\lambda}} \frac{\mathrm{e}^{-\mathrm{i} \lambda s}}{s^{p}} \mathrm{~d} s=\mathfrak{n}(p)-\beta^{1-p} \int_{1}^{\infty} \frac{\mathrm{e}^{-\mathrm{i} \beta s}}{s^{p}} \mathrm{~d} s .
$$

An integration by parts completes the argument.

Lemma 3. For any $\lambda, \alpha>0$, we have the equality

$$
\begin{aligned}
\lambda^{1-p} \int_{\alpha}^{\infty} \mathrm{e}^{-\mathrm{i} \lambda s} f(s) \mathrm{d} s= & \frac{\lambda^{1-p}}{2} \int_{\alpha}^{\alpha+\frac{\pi}{\lambda}} \mathrm{e}^{-\mathrm{i} \lambda s} f(s) \mathrm{d} s+\frac{\mathrm{i} e^{-\mathrm{i} \lambda \alpha}}{2 \lambda^{p}}\left[f\left(\alpha+\frac{\pi}{\lambda}\right)-f(\alpha)\right] \\
& -\frac{\mathrm{i}}{\lambda^{p}} \int_{\alpha}^{\infty} \mathrm{e}^{-\mathrm{i} \lambda s} f^{\prime}(s) \mathrm{d} s+\frac{\mathrm{i}}{2 \lambda^{p}} \int_{\alpha}^{\alpha+\frac{\pi}{\lambda}} \mathrm{e}^{-\mathrm{i} \lambda s} f^{\prime}(s) \mathrm{d} s .
\end{aligned}
$$

Proof. By direct calculations, we derive the identity

$$
\int_{\alpha}^{\infty} \mathrm{e}^{-\mathrm{i} \lambda s} f(s) \mathrm{d} s=\frac{1}{2} \int_{\alpha}^{\alpha+\frac{\pi}{\lambda}} \mathrm{e}^{-\mathrm{i} \lambda s} f(s) \mathrm{d} s-\frac{1}{2} \int_{\alpha}^{\infty} \mathrm{e}^{-\mathrm{i} \lambda s}\left(\int_{s}^{s+\frac{\pi}{\lambda}} f^{\prime}(\sigma) \mathrm{d} \sigma\right) \mathrm{d} s,
$$

and the conclusion follows from the Fubini theorem.

Besides, assuming (1) true for some $p \in[0,1)$, we introduce the nondecreasing function vanishing at zero

$$
\omega_{p}(s)=\sup _{t \in(0, s)}\left|t^{p} f(t)-\ell\right|
$$

We shall treat separately two cases. 
Case $\boldsymbol{p}>\mathbf{0}$. Define the function $\beta$ on $\mathbb{R}^{+}$as follows: setting $\lambda_{0}=0$, select a strictly increasing sequence $\lambda_{n} \geq n^{2}$ in such a way that

$$
\omega_{p}\left(\frac{n}{\lambda_{n}}\right) \leq \frac{1}{n^{2}}
$$

Then, put

$$
\beta(\lambda)=n \quad \text { for } \lambda \in\left[\lambda_{n}, \lambda_{n+1}\right) .
$$

By construction, the (nonnegative) function $\beta$ is nondecreasing with

$$
\lim _{\lambda \rightarrow \infty} \beta(\lambda)=\infty
$$

Besides

$$
\lim _{\lambda \rightarrow \infty} \beta(\lambda) \omega_{p}\left(\frac{\beta(\lambda)}{\lambda}\right)=0
$$

and

$$
\lim _{\lambda \rightarrow \infty} \frac{\beta(\lambda)}{\lambda}=0
$$

Indeed, if $n \geq 1$ and $\lambda \in\left[\lambda_{n}, \lambda_{n+1}\right)$, we have that

$$
\beta(\lambda) \omega_{p}\left(\frac{\beta(\lambda)}{\lambda}\right)=n \omega_{p}\left(\frac{n}{\lambda}\right) \leq n \omega_{p}\left(\frac{n}{\lambda_{n}}\right) \leq \frac{1}{n},
$$

and

$$
\frac{\beta(\lambda)}{\lambda}=\frac{n}{\lambda} \leq \frac{n}{\lambda_{n}} \leq \frac{1}{n}
$$

At this point, we write

$$
\lambda^{1-p} \hat{f}(\lambda)=\mathfrak{I}_{1}(\lambda)+\mathfrak{I}_{2}(\lambda)+\mathfrak{I}_{3}(\lambda),
$$

where

$$
\begin{aligned}
& \mathfrak{I}_{1}(\lambda)=\ell \lambda^{1-p} \int_{0}^{\frac{\beta(\lambda)}{\lambda}} \frac{\mathrm{e}^{-\mathrm{i} \lambda s}}{s^{p}} \mathrm{~d} s, \\
& \mathfrak{I}_{2}(\lambda)=\lambda^{1-p} \int_{0}^{\frac{\beta(\lambda)}{\lambda}} \mathrm{e}^{-\mathrm{i} \lambda s} \frac{s^{p} f(s)-\ell}{s^{p}} \mathrm{~d} s, \\
& \mathfrak{I}_{3}(\lambda)=\lambda^{1-p} \int_{\frac{\beta(\lambda)}{\lambda}}^{\infty} \mathrm{e}^{-\mathrm{i} \lambda s} f(s) \mathrm{d} s .
\end{aligned}
$$

- From Lemma 2 it is clear that

$$
\lim _{\lambda \rightarrow \infty} \mathfrak{I}_{1}(\lambda)=\ell \mathfrak{n}(p) .
$$

- By direct calculations, for all $\lambda \geq \lambda_{1}$,

$$
\left|\mathfrak{I}_{2}(\lambda)\right| \leq \lambda^{1-p} \omega_{p}\left(\frac{\beta(\lambda)}{\lambda}\right) \int_{0}^{\frac{\beta(\lambda)}{\lambda}} \frac{1}{s^{p}} \mathrm{~d} s \leq \frac{1}{1-p} \beta(\lambda) \omega_{p}\left(\frac{\beta(\lambda)}{\lambda}\right) \rightarrow 0
$$

as $\lambda \rightarrow \infty$. 
- From Lemma 3 with $\alpha=\frac{\beta(\lambda)}{\lambda}$, we get

$$
\begin{aligned}
\mathfrak{I}_{3}(\lambda)= & \frac{\lambda^{1-p}}{2} \int_{\frac{\beta(\lambda)}{\lambda}}^{\frac{\beta(\lambda)}{\lambda}+\frac{\pi}{\lambda}} \mathrm{e}^{-\mathrm{i} \lambda s} f(s) \mathrm{d} s+\frac{\mathrm{i} \mathrm{e}^{-\mathrm{i} \beta(\lambda)}}{2 \lambda^{p}}\left[f\left(\frac{\beta(\lambda)}{\lambda}+\frac{\pi}{\lambda}\right)-f\left(\frac{\beta(\lambda)}{\lambda}\right)\right] \\
& -\frac{\mathrm{i}}{\lambda^{p}} \int_{\frac{\beta(\lambda)}{\lambda}}^{\infty} \mathrm{e}^{-\mathrm{i} \lambda s} f^{\prime}(s) \mathrm{d} s+\frac{\mathrm{i}}{2 \lambda^{p}} \int_{\frac{\beta(\lambda)}{\lambda}}^{\frac{\beta(\lambda)}{\lambda}+\frac{\pi}{\lambda}} \mathrm{e}^{-\mathrm{i} \lambda s} f^{\prime}(s) \mathrm{d} s .
\end{aligned}
$$

We now estimate the four terms in the right-hand side. For $\lambda$ sufficiently large,

$$
\begin{aligned}
\frac{\lambda^{1-p}}{2}\left|\int_{\frac{\beta(\lambda)}{\lambda}}^{\frac{\beta(\lambda)}{\lambda}+\frac{\pi}{\lambda}} \mathrm{e}^{-\mathrm{i} \lambda s} f(s) \mathrm{d} s\right| & \leq|\ell| \lambda^{1-p} \int_{\frac{\beta(\lambda)}{\lambda}}^{\frac{\beta(\lambda)}{\lambda}+\frac{\pi}{\lambda}} \frac{1}{s^{p}} \mathrm{~d} s \\
& =\frac{|\ell|}{1-p}[\beta(\lambda)]^{1-p}\left[\left(1+\frac{\pi}{\beta(\lambda)}\right)^{1-p}-1\right],
\end{aligned}
$$

which, recalling that $\beta(\lambda) \rightarrow \infty$, converges to zero as $\lambda \rightarrow \infty$. Concerning the second term, its modulus is readily seen to be bounded by

$$
\frac{1}{2 \lambda^{p}}\left[\left|f\left(\frac{\beta(\lambda)}{\lambda}+\frac{\pi}{\lambda}\right)\right|+\left|f\left(\frac{\beta(\lambda)}{\lambda}\right)\right|\right] \sim \frac{|\ell|}{[\beta(\lambda)]^{p}} \rightarrow 0 .
$$

Finally, the two remaining terms are controlled by

$$
\frac{3}{2 \lambda^{p}} \int_{\frac{\beta(\lambda)}{\lambda}}^{\infty}\left|f^{\prime}(s)\right| \mathrm{d} s=\frac{3}{2[\beta(\lambda)]^{p}}\left(\frac{\beta(\lambda)}{\lambda}\right)^{p} \int_{\frac{\beta(\lambda)}{\lambda}}^{\infty}\left|f^{\prime}(s)\right| \mathrm{d} s \rightarrow 0
$$

on account of (2). This finishes the proof of the case $p>0$.

Case $\boldsymbol{p}=\mathbf{0}$. Extending by continuity $f$ at zero, using Lemma 3 and letting then $\alpha \rightarrow 0$ we get

$$
\begin{aligned}
\lambda \hat{f}(\lambda)= & \frac{\lambda}{2} \int_{0}^{\frac{\pi}{\lambda}} \mathrm{e}^{-\mathrm{i} \lambda s} f(s) \mathrm{d} s+\frac{\mathrm{i}}{2}\left[f\left(\frac{\pi}{\lambda}\right)-\ell\right] \\
& -\mathrm{i} \int_{0}^{\infty} \mathrm{e}^{-\mathrm{i} \lambda s} f^{\prime}(s) \mathrm{d} s+\frac{\mathrm{i}}{2} \int_{0}^{\frac{\pi}{\lambda}} \mathrm{e}^{-\mathrm{i} \lambda s} f^{\prime}(s) \mathrm{d} s .
\end{aligned}
$$

It is clear from the continuity of $f$ and the Riemann-Lebesgue lemma that the last three terms in the right-hand side above go to zero as $\lambda \rightarrow \infty$. Moreover, as $\mathfrak{n}(0)=-\mathrm{i}$,

$$
\frac{\lambda}{2} \int_{0}^{\frac{\pi}{\lambda}} \mathrm{e}^{-\mathrm{i} \lambda s} f(s) \mathrm{d} s=\ell \mathfrak{n}(0)+\frac{\lambda}{2} \int_{0}^{\frac{\pi}{\lambda}} \mathrm{e}^{-\mathrm{i} \lambda s}[f(s)-\ell] \mathrm{d} s,
$$

and

$$
\left|\frac{\lambda}{2} \int_{0}^{\frac{\pi}{\lambda}} \mathrm{e}^{-\mathrm{i} \lambda s}[f(s)-\ell] \mathrm{d} s\right| \leq \frac{\pi}{2} \omega_{0}\left(\frac{\pi}{\lambda}\right) \rightarrow 0
$$

when $\lambda \rightarrow \infty$. 


\section{Application to Equations with Memory}

In this second part, we discuss an application to equations with memory. To this end, let $A$ be a strictly positive unbounded linear operator on a separable real Hilbert space, with inverse $A^{-1}$ not necessarily compact. We consider the linear equation with memory

$$
\partial_{t t} u(t)+A u(t)+\int_{0}^{\infty} \mu(s)[u(t)-u(t-s)] \mathrm{d} s=0,
$$

where $u(0)$ and $\partial_{t} u(0)$, as well as the past history $u(-s)_{\mid s>0}$, are assigned initial data. The nonnegative function $\mu \not \equiv 0$, called memory kernel, is supposed to be nonincreasing, absolutely continuous and summable on $\mathbb{R}^{+}$(hence $\mu^{\prime}$ is summable at infinity). A concrete version of the equation, where $A$ is a particular realization of the Laplacian, arises in the theory of hereditary electromagnetism, and serves as a model for the evolution of the electromagnetic field in the ionosphere (see [7] for more details).

The well-posedness of the related Cauchy problem and the asymptotic behavior of the solutions have been studied in $[7,10]$. There, by introducing in the same spirit of [4] the auxiliary "memory variable", the equation is shown to generate a contraction semigroup $S(t)=\mathrm{e}^{t \mathbb{A}}$ acting on a suitable Hilbert space accounting for the presence of the memory. Besides, such a semigroup fails to be exponentially stable. Nevertheless, if the inclusion $i \mathbb{R} \subset \rho(\mathbb{A})$ holds $^{1}$, in the light of the works of Batty and coauthors $[1,2]$ the semigroup $S(t)$ turns out to be semiuniformly stable, i.e.

$$
\lim _{t \rightarrow \infty}\left\|S(t) \mathbb{A}^{-1}\right\|=0 .
$$

In addition, the very same calculations needed to prove the lack of exponential stability provide an estimate from below of the norm of the resolvent operator $(i \lambda-\mathbb{A})^{-1}$. Indeed, the following result holds (see $[5,9]$ ).

Theorem 4. Assume that $\mu$ satisfies for some $\delta>0$ the classical condition [4]

$$
\mu^{\prime}(s)+\delta \mu(s) \leq 0, \quad \forall s \in \mathbb{R}^{+} .
$$

Then $\mathrm{i} \mathbb{R} \subset \rho(\mathbb{A})$, implying that $S(t)$ is semiuniformly stable. Besides,

$$
\limsup _{\lambda \rightarrow \infty} \frac{|\hat{\mu}(\lambda)|}{\lambda}\left\|(\mathrm{i} \lambda-\mathbb{A})^{-1}\right\|>0
$$

where $\hat{\mu}$ is the half Fourier transform of $\mu$.

The latter estimate turns out to be a crucial tool in establishing the optimal decay rate, for it is known from [2] that if

$$
\limsup _{\lambda \rightarrow \infty} \lambda^{-\alpha}\left\|(\mathrm{i} \lambda-\mathbb{A})^{-1}\right\|>0
$$

for some $\alpha>0$, then $\left\|S(t) \mathbb{A}^{-1}\right\|$ cannot decay faster than $t^{-\frac{1}{\alpha}}$. Thus, the problem of finding sharp polynomial growth rates at infinity of $\hat{\mu}$ becomes paramount. As an immediate consequence of Theorem 1, we have a notable "quantitative version" of Theorem 4.

\footnotetext{
${ }^{1} \rho(\mathbb{A})$ denotes the resolvent set of the (complexification of the) infinitesimal generator $\mathbb{A}$.
} 
Theorem 5. Within the hypotheses of Theorem 4, assume there exist $p \in[0,1)$ and $\ell>0$ such that

$$
\lim _{s \rightarrow 0} s^{p} \mu(s)=\ell
$$

Then

$$
\limsup _{\lambda \rightarrow \infty} \lambda^{p-2}\left\|(i \lambda-\mathbb{A})^{-1}\right\|>0 .
$$

Accordingly, $\left\|S(t) \mathbb{A}^{-1}\right\|$ cannot decay faster than $t^{-\frac{1}{2-p}}$.

Remark. In $[5,9]$ it is also shown that if the first limit above occurs for $p=0$, then $\left\|S(t) \mathbb{A}^{-1}\right\|$ decays at least as $t^{-\frac{1}{2}}$. In which case, we conclude from Theorem 5 that $t^{-\frac{1}{2}}$ is actually the optimal decay rate.

The fact that Theorem 1 has not been considered in the earlier PDE literature is exemplified, for instance, by the works [5, 6, 8, 9], where the above quantitative estimates are carried out only for particular kernels of the form

$$
\mu(s)=s^{-p} \mathrm{e}^{-\delta s},
$$

with $\delta>0$ and $p \in[0,1)$, for which $\hat{\mu}$ can be explicitly computed.

\section{REFERENCES}

[1] W. Arendt and C.J.K. Batty, Tauberian theorems and stability of one-parameter semigroups, Trans. Amer. Math. Soc. 306 (1988), 837-852.

[2] C.J.K. Batty and T. Duyckaerts, Non-uniform stability for bounded semi-groups on Banach spaces, J. Evol. Equ. 8 (2008), 765-780.

[3] N.H. Bingham, C.M. Goldie and J.L. Teugels, Regular variation, John Wiley \& Sons, New York, 1971.

[4] C.M. Dafermos, Asymptotic stability in viscoelasticity, Arch. Rational Mech. Anal. 37 (1970), 297308.

[5] V. Danese and F. Dell'Oro, Lack of exponential stability for a class of second-order systems with memory, Proc. Roy. Soc. Edinburgh Sect. A (in press).

[6] H.D. Fernández Sare, J.E. Muñoz Rivera and R. Quintanilla, On the rate of decay in interacting continua with memory, J. Differential Equations 251 (2011), 3583-3605.

[7] C. Giorgi, M.G. Naso and V. Pata, Energy decay of electromagnetic systems with memory, Math. Models Methods Appl. Sci. 15 (2005), 1489-1502.

[8] M. Grasselli, J.E. Muñoz Rivera and M. Squassina, Asymptotic behavior of a thermoviscoelastic plate with memory effects, Asymptot. Anal. 63 (2009), 55-84.

[9] J.E. Muñoz Rivera and M.G. Naso, Optimal energy decay rate for a class of weakly dissipative second-order systems with memory, Appl. Math. Lett. 23 (2010), 743-746.

[10] J.E. Muñoz Rivera, M.G. Naso and E. Vuk, Asymptotic behavior of the energy for electromagnetic systems with memory, Math. Methods Appl. Sci. 27 (2004), 819-841.

[11] D.V. Widder, The Laplace Transform, Princeton University Press, Princeton, NJ, 1941.

Politecnico di Milano - Dipartimento di Matematica

Via Bonardi 9, 20133 Milano, Italy

E-mail address: filippo.delloro@polimi.it (F. Dell'Oro)

E-mail address: enrico.laeng@polimi.it (E. Laeng)

E-mail address: vittorino.pata@polimi.it (V. Pata) 